\title{
INFLUENCE OF ETRETINATE ON BLOOD AND LIVER IN RATS
}

\author{
Koji SAKAMOTO, Takako KASAHARA, Sadao NAKAYAMA, \\ Yasumitsu NAKAl* and Jun'ichi KUDO* \\ Department of Pharmacology. "Department of Anatomy. School of Medicine, \\ Showa University, Tokyo 142. Japan
}

Accepted July 12, 1982

A comparative toxicological study was carried out for etretinate and retinyl palmitate. a naturally occurring retinol, using rats. Hematological and blood biochemical examinations revealed that etretinate showed lower toxicity and a larger difference between its clinically effective dose and hypervitaminosis A-causing dose, compared with retinyl palmitate. Moreover, electron microscopy of the liver demonstrated a marked accumulation of lipid droplets in the cytoplasm of fatstoring cells treated with retinyl palmitate, suggesting that etretinate may be more rapidly metabolized than retinyl palmitate in the liver of the animal.

It is well known that retinoic acid and its derivatives (retinoids) at their clinically effective doses cause hypervitaminosis A which is an adverse effect of the substances (1). Also, it has been reported that hypervitaminosis $A$ induced by treatment with various retinoids is considered to be due to their tendency to accumulate in the liver. Such retinoids are finally stored in the liver in the form of retinyl palmitate (2). On the other hand, etretinate is effective for many kinds of dermal diseases and less toxic than natural retinoids. The present report deals with blood and liver levels of etretinate and retinyl palmitate in rats immediately after administration and during their recovery periods, in addition to hematological findings and electron micrographs of the liver. A short discussion is also presented. The test substances, etretinate (ethyl-all-trans-9-(4- metoxy-2,3,6-trimethylphenyl) -3,7-dimethyl$2,4,6,8$-nonatetraenoate) and retinyl palmitate, were kindly supplied by Nippon Roche K.K.

Sprague-Dawley male rats, 5 weeks of age. weighing about $140 \mathrm{~g}, 5$ in a group. were treated with etretinate or retinyl palmitate at a dose of $5 \mathrm{mg} / \mathrm{kg}$ or $5.75 \mathrm{mg} / \mathrm{kg}$. respectively, by oral gavage once a day for 4 weeks. Another 2 groups, each consisting of 5 animals, were given nothing or the peanut oil vehicle only. Animals were sacrificed after collecting blood samples $24 \mathrm{hr}$ or 2 weeks after the 4-week administration period.

Hematological examinations were carried out for erythrocyte counts (RBC), leukocyte counts (WBC) (automatic blood corpuscle counter). hemoglobin ( $\mathrm{Hb}$ ) (cyanomethemoglobin method) and hematocrit $(\mathrm{Ht})$ (capillary method). Serum biochemical examinations were done for total protein, A/G ratio, GOT, GPT, LDH, alkaline posphatase, bilirubin, creatinine, uric acid, BUN, total cholesterol (autoanalyzer. Hitachi 716. Hitachi), triglycerides, phospholipids (autoanalyzer, Clinalyzer 6ch. JEOL) and nonesterified fatty acids (enzyme method). The serum and liver levels of retinoids were assessed by measuring the corresponding peak heights on HPLC charts. The serum levels of retinoids were determined as retinol.

Prior to liver excision, the animals were fixed by slow perfusion of a fixative of $1.5 \%$ glutaraldehyde buffered to $\mathrm{pH} 7.4$ with 0.06 


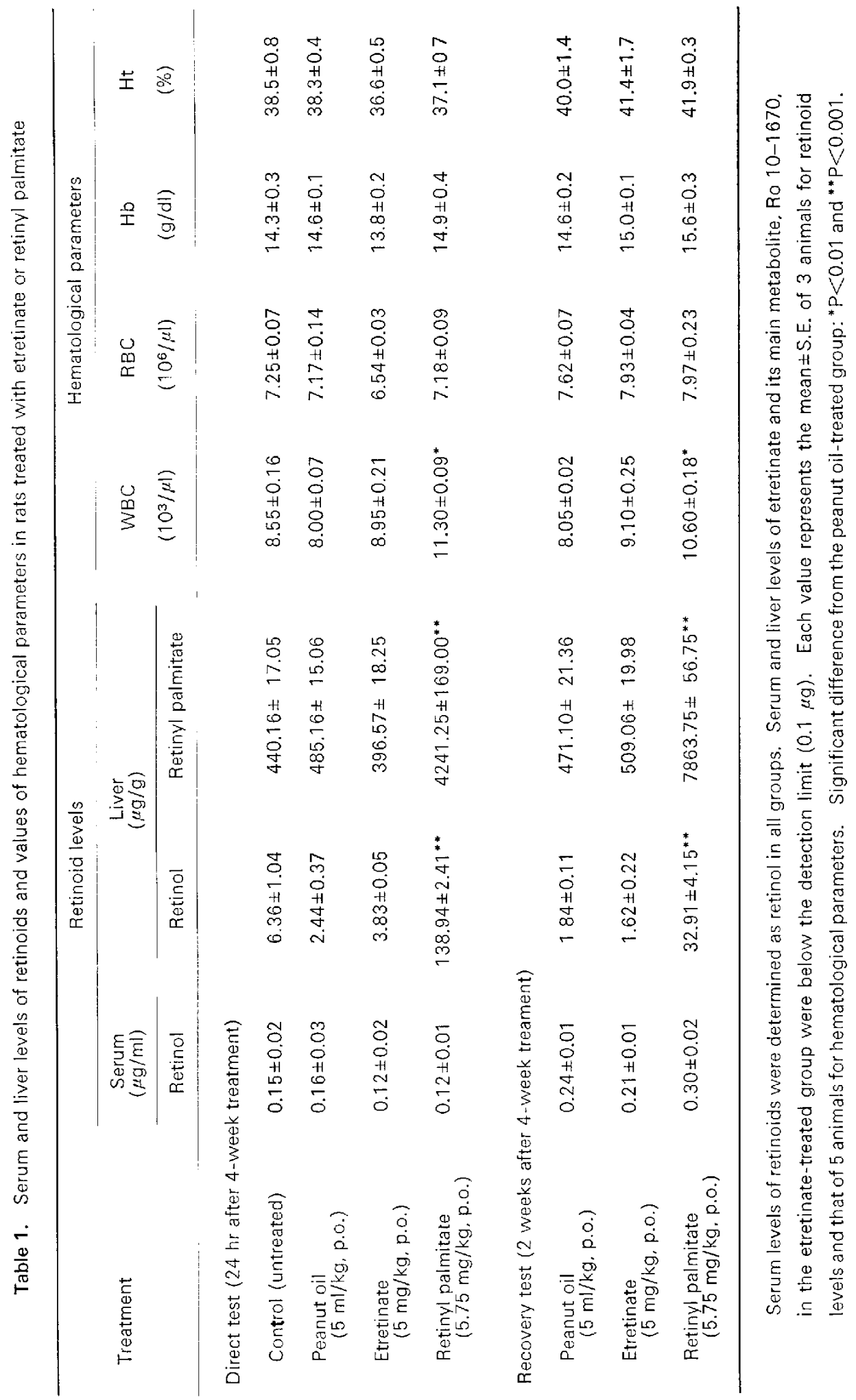


M cacodylate through the portal vein. The liver was cut into small pieces and postfixed with 1\% osmium tetroxide buffered with $0.1 \mathrm{M}$ phosphate $(\mathrm{pH} 7.4)$. After dehydration in a graded ethanol series and propylene oxide, the tissue pieces were embedded in Epon 812. Thin sections that were double-stained with uranyl acetate and lead citrate were examined by an electron microscope ( $H U-12$. Hitachi).

Etretinate and retinyl palmitate exerted no influence on body weights and weights of organs such as the liver, kidney, spleen and eye-bail. Etretinate had no effect on any parameters in hematological and serum biochemical examinations. On the contrary, administration of retinyl palmitate resulted in an increase in WBC $24 \mathrm{hr}$ after the termination of treatment and high values even during the recovery period, showing no change in the other parameters (Table 1)

There was little difference in serum levels of retinol and liver levels of retinol and retinyl palmitate, both $24 \mathrm{hr}$ and 2 weeks after the 4-week treatment period, between the etretinate group and the vehicle control or the untreated control group. Etretinate and its main metabolites, one of which was Ro 10-1670, were detected in neither serum nor liver, both $24 \mathrm{hr}$ and 2 weeks after the 4 -week etretinate administration. Twenty-four hours after retinyl palmitate administration, there was no difference between the serum leve of retinol in the untreated group and the vehicle control group, while the liver levels of retinol and retinyl palmitate were markedly increased. After 2 weeks, the liver level of retinol was decreased, while that of retinyl palmitate was further increased to about 1.8 times as high as the level after $24 \mathrm{hr}$ (Table 1)

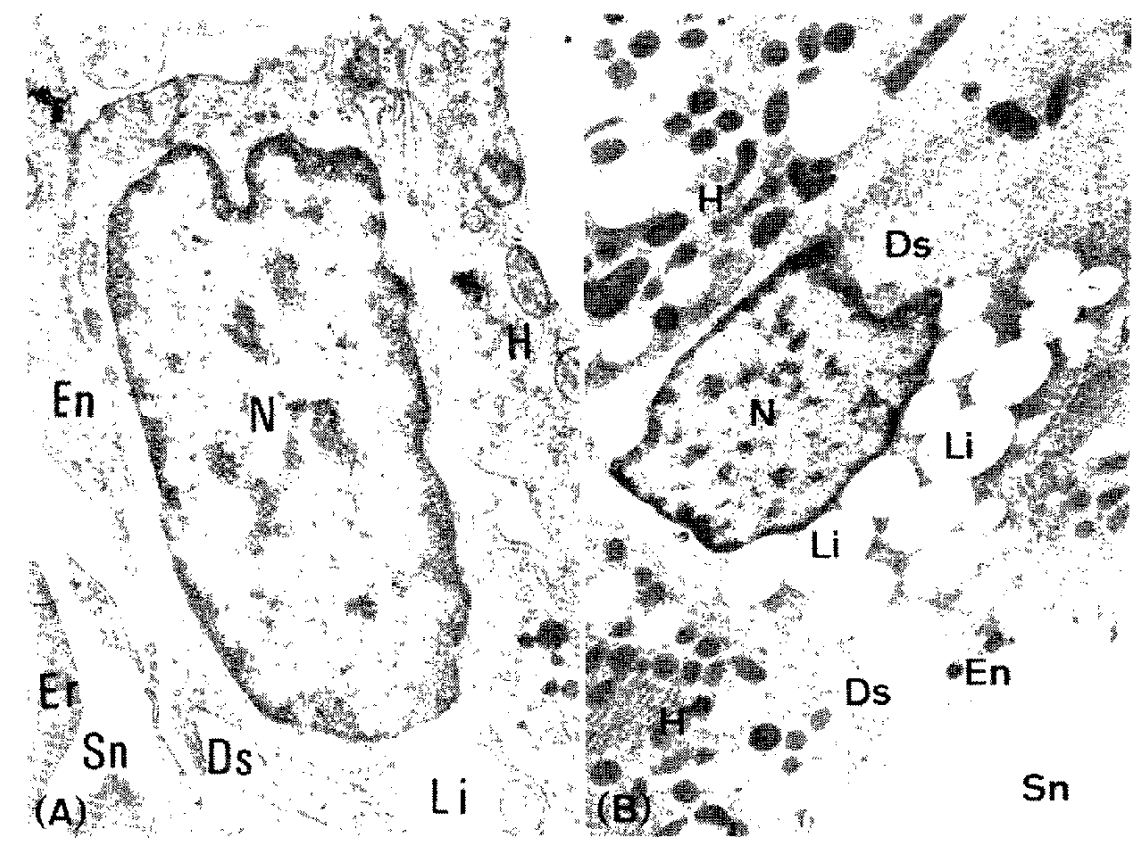

Fig. 1. Electron micrograph of the rat liver excised $24 \mathrm{hr}$ after 4 -week daily administration of retinoids. A: A fat sturing ce!! (N) in the Disse's space of the liver of the animal treated whth etretınte contains several ipid droplets (Li) in the cytoplasm. N: fat storing cell nucleus. Sn: sinusoid. En: onootholial lining. Er: erythrocytes, H: hopatocytes, $\times 12,000$. B: Lipid droplets ( $\mathrm{Li}$ ) in a fat-storing cell (N) of the liver of the animal treated with rotinyl palmitate were markedly increased in number. N: fat-storing cell nucleus. Sn: sinusoid, En: erdotnelial linıng. Ds: Disse's space, $H$ : hepatocytes. $\times 6,000$ 
There were several lipid droplets observed in the cytoplasm of fat-storing cells in the Disse's space of the liver prepared $24 \mathrm{hr}$ after the 4-week etretinate treatment period. The picture was similar to that of the untreated controf or the vehicle control livers (not published). Twenty-four hours aftèr retinyl palmitate administration. many droplets were observed in fat-storing cells. There was no change in the fine structure of cell organelles or nuclei of fat-storing cells by the administration of either etretinate or retinyl palmitate (Fig. 1).

Concerning existing retinoids, it is known that as they tend to accumulate in the liver, hypervitaminosis A appears before the blood level of the retinols reaches a clinically effective one (1). On the other hand, it is reported that hypervitaminosis A does not occur with etretinate at the clinically effective doses for various skin keratoses. As a matter of fact, the therapeutic ratio (the ratio of the incidence rate of hypervitaminosis A to ED50 for papilloma) with etretinate is $10 \%$ of that with retinoic acid (3). Mahadevan et al. have reported the accumulation of natural retinoids as the test results with various retinyl esters and retinol. According to them, the administered retinoids accumulated in the liver eventually as retinyl palmitate, and the liver level of retinyl palmitate was higher at $24 \mathrm{hr}$ post-dose than immediately after administration even in the group receiving retinyl palmitate (2).

In the present study, it was inferred from hematological findings that etretinate was lower in toxicity than retinyl palmitate. The determination of the serum and liver levels of retinoids following administration of etretinate or retinyl palmitate revealed that etretinate was metabolized more rapidly than retinyl palmitate, and the accumulation of etretinate in the liver was very low. The liver level of retinyl palmitate was higher in the 2 nd week than at 24th hour after the 4-week retinyl palmitate treatment period. suggesting a high accumulation.

The difference in the accumulation in the liver between etretinate and retinyl palmitate was also demonstrated by electron microscopy. There was almost no accumulation of lipid droplets in the fat-storing cells of the liver by etretinate administration, while treatment with retinyl palmitate resulted in an apparent increased number of lipid droplets which might contain retinyl palmitate.

Conjecturing from the extremely low accumulation of etretinate in the liver, it can be concluded that the rapid metabolizability of etretinate contributes to the manifestation of lower toxicity such as the small incidence of hypervitaminosis $\mathrm{A}$ at the clinically effective dose of etretinate than that of naturally occurring retinoids.

\section{REFERENCES}

1) Bollag, W.: From vitamin $A$ to retinoids: chemical and pharmacologica! aspects. In Retinoids, Edited by Orfanos, E.C., Grupper, Ch., Polano, M.K.. Schuppli, R., Braun-Falco, O. and Farber, E.M.. p. 5-11, Springer-Verlag. Berlin. Heidelberg. New York (1980)

2) Mahadevan, S, Deshmukh, D.S. and Ganguly, J.: The fate of intracardially administered retinyl esters in the rat. Biochem J. 93, 499-503 (1964)

3) Bollag, W.: Therapeutic effects of an aromatic retinoic acid analogue on chemically induced skin papillomas and carcinomas of mice. Eur. J. Cancer 10,731-737 (1974) 